\title{
Consumo de energía térmica y emisiones de dióxido de carbono en la fabricación de baldosas cerámicas Análisis de las industrias Española y Brasileña
}

\author{
E. MONFORT (1), A. MEZQUITA ${ }^{(1)}$, E. VAQUER ${ }^{(1)}$, G. MALLOL $^{(1)}$, H. J. $\operatorname{ALVES}^{(2)}$, A. O. BOSCHI ${ }^{(2)}$ \\ (1) Instituto de Tecnología Cerámica (ITC). Asociación de Investigación de las Industrias Cerámicas (AICE). Universitat Jaume I. Castellón. España. \\ (2) Laboratorio de Revestimientos Cerámicos (LaRC). Universidad Federal de San Carlos. Brasil
}

España y Brasil son dos de los mayores productores de baldosas cerámicas en el mundo. El proceso de fabricación de baldosas consume gran cantidad de energía térmica, y en estos dos países se obtiene principalmente de la combustión de gas natural, lo que conlleva la emisión a la atmósfera de $\mathrm{CO}_{2}$, gas de efecto invernadero.

El objetivo del trabajo presentado es mostrar comparativamente los consumos de energía térmica y las emisiones de $\mathrm{CO}_{2}$ en el proceso de obtención de baldosas cerámicas en España y en Brasil, atendiendo a las diferentes tecnologías de producción y a los distintos productos fabricados.

El consumo energético y las emisiones de $\mathrm{CO}_{2}$ son muy parecidos en ambos países, en el proceso de obtención de baldosas por vía húmeda. En el proceso vía seca utilizado en Brasil, se consume menos energía térmica, y se emite menos $\mathrm{CO}_{2}$, que en el proceso vía húmeda, pero es un proceso que se utiliza únicamente en la obtención de un tipo de producto en particular, que presenta algunas limitaciones técnicas. En España, el uso de sistemas de cogeneración en la etapa de atomización incrementa notablemente la eficiencia energética global del proceso.

Los consumos energéticos medios de las diferentes etapas del proceso se sitúan, en ambos países, dentro del intervalo indicado en el Documento de Referencia sobre las Mejores Técnicas Disponibles en la Industria Cerámica de la Unión Europea (BREF de la Industria Cerámica).

Palabras clave: consumo energético, emisiones de $\mathrm{CO}_{2}$, baldosas cerámicas, España, Brasil

Thermal energy consumption and carbon dioxide emissions in ceramic tile manufacture - Analysis of the Spanish and Brazilian industries

Spain and Brazil are two of the world's biggest ceramic tile producers. The tile manufacturing process consumes a great quantity of thermal energy that, in these two countries, is mainly obtained from natural gas combustion, which entails $\mathrm{CO}_{2}$ emission, a greenhouse gas.

This study presents a comparative analysis of the thermal energy consumption and $\mathrm{CO}_{2}$ emissions in the ceramic tile manufacturing process in Spain and Brazil, in terms of the different production technologies and different products made. The energy consumption and $\mathrm{CO}_{2}$ emissions in ceramic tile manufacture by the wet process are very similar in both countries. In the dry process used in Brazil, less thermal energy is consumed and less $\mathrm{CO}_{2}$ is emitted than in the wet process, but it is a process that is only used in manufacturing one particular type of product, which exhibits certain technical limitations. While in Spain the use of cogeneration systems in spray-dryers improves significantly the global energy efficiency.

The average energy consumption in the different process stages, in both countries, lies within the range indicated in the Reference Document on Best Available Techniques in the Ceramic Manufacturing Industry (BREF of the Ceramic Manufacturing Industry) of the European Union.

Keywords: energy consumption, $\mathrm{CO}_{2}$ emissions, ceramic tiles, Spain, Brazil

\section{INTRODUCCIÓN}

La producción mundial de baldosas cerámicas prácticamente se ha duplicado en los últimos diez años, y países como España y Brasil destacan por su contribución a este crecimiento, trabajando principalmente en el desarrollo de productos diferenciados y en la mejora de los procesos productivos. En la última década España y Brasil han estado siempre entre los principales países productores de baldosas cerámicas. Actualmente, Brasil es el segundo productor mundial y también el segundo mayor consumidor de baldosas del mundo. España es el tercer país del mundo exportador de baldosas y destaca por producir productos de elevado valor añadido [1].

Como consecuencia de la expansión del sector la demanda de combustibles para la generación de energía térmica, utilizada en varias etapas del proceso productivo (secado, atomización y cocción), ha aumentado significativamente, 
siendo un indicador directo del aumento de la producción. El combustible más utilizado por las industrias de baldosas cerámicas para la generación de energía térmica es el gas natural, combustible fósil cuya combustión produce la emisión de dióxido de carbono $\left(\mathrm{CO}_{2}\right)$. El $\mathrm{CO}_{2}$ es uno de los gases de efecto invernadero responsables del cambio climático y del calentamiento global del planeta. Las emisiones de $\mathrm{CO}_{2}$ en el proceso de fabricación de baldosas tienen dos orígenes: 1) Emisiones de combustión, que son las emisiones producidas durante la reacción exotérmica de combustión entre el combustible y el comburente; 2) Emisiones de proceso, que son las emisiones originadas durante la etapa de cocción por la descomposición de los carbonatos presentes en las materias primas, y por la descomposición de la materia orgánica presente en las piezas, tanto en el soporte como en las aplicaciones serigráficas.

El coste de la energía térmica es uno de los costes productivos más significativos en la fabricación de baldosas cerámicas, y las emisiones de $\mathrm{CO}_{2}$ resultantes de esta actividad industrial son significativas $[2,3]$. Sin embargo, existen pocos datos disponibles sobre el consumo de energía térmica en la fabricación de baldosas cerámicas teniendo en cuenta las particularidades del proceso de fabricación, los distintos equipos, las condiciones de operación y las características de las materias primas, datos que sirven de base para estimar con mayor precisión las emisiones de $\mathrm{CO}_{2}$. El conocimiento por parte de las industrias del consumo térmico involucrado en la fabricación de baldosas es primordial, pues permite tomar medidas correctivas relacionadas con las condiciones de operación de los equipos cuando son necesarias, se evitan pérdidas y permite tomar las decisiones más adecuadas sobre la compra de equipos, buscando el uso más eficiente de la energía, una reducción de costes y un mayor control sobre las emisiones de $\mathrm{CO}_{2}$.

Las emisiones de gases de efecto invernadero son objeto de estudio y control a nivel internacional. En España, la legislación vigente se basa en las directivas europeas sobre el sistema de comercio de emisiones [4 y 5]. En Brasil, todavía no existe una ley similar a la europea en la que puedan ser incluidas las industrias del sector cerámico.

Este artículo presenta los resultados de un trabajo experimental realizado por técnicos del Laboratorio de Revestimientos Cerámicos y del Instituto de Tecnología Cerámica en empresas de Brasil y España, respectivamente, que incluye el consumo energético de los hornos, secaderos y atomizadores de la industria de fabricación de baldosas cerámicas. Los datos obtenidos se han tratado y presentado según el tipo de producto producido, las características de los equipos, las etapas de los procesos productivos y las tecnologías de fabricación. Los resultados presentados constituyen una base de datos inédita sobre el consumo térmico y las emisiones de $\mathrm{CO}_{2}$ inherentes al proceso de fabricación de baldosas cerámicas, que revela las peculiaridades de cada país.

A continuación se describen brevemente los perfiles de los sectores de fabricación de baldosas cerámicas español y brasileño, además de las principales características de los productos fabricados.

El sector español de fabricación de baldosas cerámicas, desde el año 2000 hasta 2008, produjo una media anual de 600 millones de $\mathrm{m}^{2}$. A partir de 2008, la situación de crisis financiera internacional propició un descenso de la producción que alcanzó su valor mínimo en el año 2009. En la figura 1 se muestra la evolución de la producción nacional de baldosas cerámicas en España desde el año 2000 hasta 2010 [1].

Los datos correspondientes al sector español mostrados en este artículo corresponden al año 2008. En el año 2008, el sector español de fabricación de baldosas cerámicas estaba constituido por más de 215 empresas, de las cuales 13 eran productoras de polvo atomizado. En el proceso productivo utilizado mayoritariamente la preparación de las materias primas se realiza vía húmeda, el conformado de las baldosas se realiza por prensado, y la cocción del soporte y del esmalte se efectúa de manera conjunta, por monococción.

La localización de las empresas se sitúa principalmente en la Comunitat Valenciana, donde están ubicadas el $87 \%$ del total, con una cuota de producción sobre el total nacional de aproximadamente el 95\%. En la Comunitat Valenciana, el mayor número de empresas está en la provincia de Castellón.

En el año 2008, el sector español de baldosas cerámicas produjo 495 millones de metros cuadrados, por valor de cerca de 3.700 millones de euros. Cerca del 58\% de la producción se destina a la exportación, a 180 países.

Los productos fabricados principalmente por el sector cerámico español de baldosas comprenden: azulejos, que son baldosas que presentan una elevada absorción de agua $(>10 \%)$, utilizadas para el revestimiento de paredes, gres esmaltado, que tiene una absorción de agua menor que el azulejo $(<3 \%)$, también denominado pavimento, adecuado para suelos tanto interiores como exteriores, y gres porcelánico, producto con una muy baja absorción de agua $(<0,5 \%)$, que se utiliza tanto para suelos como para fachadas, interiores o exteriores, y se fabrica tanto pulido como sin pulir, esmaltado y sin esmaltar.

Tanto los azulejos como los productos gresificados pueden obtenerse a partir de arcillas de coloración roja o blanca en cocido. Durante el año 2008, el $41 \%$ de la producción fueron azulejos, el 35\% gres esmaltado, y casi el 20\% de la producción fueron baldosas de gres porcelánico.

Las emisiones totales de dióxido de carbono originadas en el proceso de fabricación de baldosas cerámicas se estiman, en 2008, en 2,889 millones de toneladas de $\mathrm{CO}_{2}$, de las cuales $0,277(9,6 \%)$ proceden de la descomposición de los carbonatos presentes en las composiciones de azulejos y de gres, y el 2,612 restantes proceden de la combustión del gas natural, principal combustible utilizado en España en la fabricación de baldosas cerámicas.

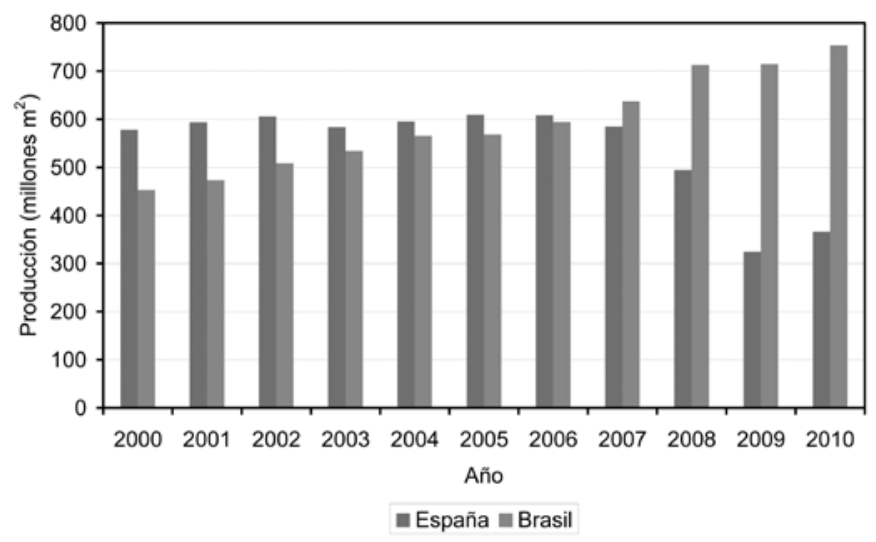

Figura 1. Evolución de la producción de baldosas cerámicas en España y Brasil en la última década. 
Actualmente, la legislación española que regula las emisiones de gases de efecto invernadero está basada en la legislación europea, en vigor desde 2003. Esta legislación afecta actualmente a menos del $20 \%$ de las empresas, pero a partir de 2013 la normativa va a cambiar, y pasarán a estar afectadas por el régimen europeo del comercio de emisiones casi el 60\% de las instalaciones [5 y 6].

Ante esta situación, es necesario disponer de una base de datos de consumos energéticos y emisiones de $\mathrm{CO}_{2}$ para conocer la situación actual del sector y las posibilidades de aumentar la eficiencia energética del proceso.

Una característica peculiar de la industria del sector de fabricación de baldosas cerámicas brasileño, es que las industrias utilizan dos vías de procesado distintas para la fabricación de sus productos: "vía húmeda" y "vía seca", siendo el $69 \%$ de sus productos fabricados por vía seca y el $31 \%$ por vía húmeda [7].

La evolución de la producción de baldosas cerámicas en Brasil puede observarse en la figura 1. En este caso, se han tomado para realizar el estudio los datos disponibles del último (2009), por tratarse de un año suficientemente representativo de la situación habitual de funcionamiento de esta industria en Brasil [1].

En el año 2009 Brasil produjo 495,4 millones de $\mathrm{m}^{2}$ por vía seca (100\% monococción roja) y 219,5 millones de $\mathrm{m}^{2}$ por vía húmeda (5,5\% gres rojo, 22\% azulejo blanco, 22\% porcelánico y 50,5\% monococción blanca), con una producción total anual de 714,9 millones de $\mathrm{m}^{2}$. Las zonas cerámicas de Brasil se concentran en dos regiones: Criciúma (Santa Catarina) y Santa Gertrudes (São Paulo), encontrándose localizadas la mayoría de las industrias con procesado por vía seca en la región de Santa Gertrudes. Los productos obtenidos por vía seca, en general, son baldosas cerámicas esmaltadas que presentan una absorción de agua entre el 6 y el 10\% y soportes de coloración roja en cocido. El procesado del material por vía seca se caracteriza por la preparación de la composición cerámica por molienda en seco ( $\sim 5 \%$ de humedad), prensado ( $\sim 9 \%$ de humedad) y monococción rápida (20 - $30 \mathrm{~min}$.).

La composición cerámica preparada por vía seca destaca tanto por presentar una formulación típica, como por la forma como es molida. La composición está formada, en general, por sólo una materia prima constituida naturalmente por las proporciones de minerales necesarios para la fabricación de los productos deseados. Se trata, pues, de una composición formada habitualmente por una única arcilla, y en algunas ocasiones, por la mezcla de varias arcillas. La arcilla en bruto extraída de los depósitos (en bloques) es cargada en camiones y trasportada para secarla al natural en eras, donde se produce una reducción de la humedad (hasta valores próximos al
5\%), la homogeneización y el desmenuzamiento. Después del trabajo en la era, el material pasa a la molienda. En la molienda seca, la materia prima pasa en primer lugar por el molino primario (molino de martillos), y luego, la fracción más dura que no se ha conseguido molturar por el mismo se transporta a un molino secundario (molino pendular). El polvo resultante es humedecido ( 9\%) y granulado. Las siguientes etapas del proceso son prácticamente idénticas a las del proceso por vía húmeda.

Debido a las exigencias de la agenda ambiental que Brasil tiene que cumplir, es muy importante conocer el perfil de emisiones de $\mathrm{CO}_{2}$ de cada sector industrial para establecer metas para el futuro. Se estima que el país presentará un crecimiento del 4 al $7 \%$ de su producto interior bruto (PIB) en 2020. Sin embargo, el objetivo de reducir las emisiones de $\mathrm{CO}_{2}$ es del 39\% a nivel nacional, teniendo en cuenta los niveles de emisión del año 2005. En el estado de São Paulo (cuya producción de baldosas cerámicas es superior al 70\% de la producción nacional) se tiene como objetivo la reducción las emisiones de $\mathrm{CO}_{2}$ en un $20 \%$ para el año 2020.

Según los datos disponibles, el sector de las baldosas cerámicas ha presentado en los últimos diez años un crecimiento importante (incremento de cerca del 70\% de la producción nacional en la última década) y las perspectivas para los próximos años son todavía más positivas. Siendo así, el crecimiento de la producción de baldosas cerámicas en Brasil deberá impulsar la demanda de gas natural en los próximos años, y en consecuencia, aumentarán las emisiones de $\mathrm{CO}_{2}$ derivadas de la utilización de este combustible. En vista de las previsiones de crecimiento y de la necesidad de una mayor eficiencia en el uso del gas natural por las industrias, el desarrollo del sector debe realizarse junto con las menores emisiones de $\mathrm{CO}_{2}$ posibles.

A pesar de la urgencia de este tema, hasta el momento no existen datos disponibles sobre las emisiones de $\mathrm{CO}_{2}$ asociadas al sector de las baldosas cerámicas en Brasil, por lo que se consideró necesario llevar a cabo este estudio con datos cualitativos y cuantitativos.

\section{OBJETO Y ALCANCE DEL ESTUDIO}

La recopilación de información necesaria para realizar el trabajo en España y Brasil se llevó a cabo utilizando la misma metodología (definida seguidamente en el apartado 3), aplicada en un número tal de empresas que suponen un porcentaje de producción representativo del total producido en cada país en los años 2008 y 2009. La selección de las empresas en las cuales se realizaría el trabajo se hizo de manera

TABLA I. Muestra de EMPRESAS estudiadas.

\begin{tabular}{|c|c|c|c|}
\hline & & España & Brasil \\
\hline \multicolumn{2}{|c|}{ Año } & 2008 & 2009 \\
\hline \multicolumn{2}{|c|}{ Número de empresas participantes } & 55 & $10^{*}$ \\
\hline \multicolumn{2}{|c|}{ Número de plantas productivas participantes } & 65 & 14 \\
\hline \multirow{2}{*}{ Cuota de producción } & Atomizado & $48 \%$ & $35 \%$ \\
\hline & Baldosas & $34 \%$ & $20 \%$ \\
\hline
\end{tabular}

* Cinco de las diez empresas brasileñas producen baldosas por vía seca 
que permitiera analizar el sector de las baldosas cerámicas de cada país considerando sus principales características, tales como la capacidad de producción, la tecnología de producción (vía seca o vía húmeda), los tipos de productos y características de los equipos consumidores de energía, entre otros. En la tabla I se presenta un detalle de la muestra de empresas estudiadas en cada país. Se han visitado en total 65 empresas, y se han analizado 130 equipos. Los porcentajes de producción alcanzados se consideran adecuados para obtener datos representativos de la situación del sector de las baldosas cerámicas de Brasil y de España, con respecto al consumo energético y a las emisiones de $\mathrm{CO}_{2}$.

\section{METODOLOGÍA}

Inicialmente, se visitaron todas las empresas con el objetivo de conocer las instalaciones, verificar las condiciones de operación de los equipos consumidores de energía y analizar la posición de los equipos medidores de los caudales de gases calientes y de gas natural. Asimismo, se revisó si era necesario realizar acciones correctivas en la posición de los medidores de caudal para garantizar la precisión de las medidas, y se explicó a las empresas los datos generales sobre los equipos y los productos que eran necesarios para llevar a cabo el trabajo.

Las medidasexperimentales en las instalacionesindustriales se realizaron mientras el régimen de funcionamiento era estacionario, es decir, durante la duración de las medidas no se produjeron modificaciones a lo largo del tiempo de las variables de funcionamiento.

Se llevaron a cabo sucesivas mediciones de presión, caudal y temperatura, cuando el régimen de funcionamiento era estacionario, durante un determinado período de tiempo en los puntos pre-establecidos de las conducciones, ubicados en regiones que precedían a la entrada de gas natural a los equipos consumidores de energía. Los datos obtenidos fueron tratados y corregidos (a condiciones normales de presión y temperatura, y la ecuación de los gases ideales), permitiendo conocer el consumo térmico de cada equipo evaluado y hacerlos comparativos. De acuerdo con las informaciones sobre el producto, el volumen de producción y las condiciones de funcionamiento de los equipos se creó una base de datos sobre la energía térmica consumida en la fabricación de baldosas cerámicas, siendo posible establecer comparaciones entre el consumo de diferentes tipos de productos, etapas del proceso de producción, distintas vías de proceso, equipos con características constructivas diferenciadas, etc. La unidad utilizada para expresar el consumo térmico específico en las situaciones abordadas fue $\mathrm{kWh} / \mathrm{t}$ de producto cocido, que permite determinar la cantidad de energía involucrada durante el procesado de cierta cantidad de masa de material producido. De esta forma, ha sido posible comparar el consumo térmico de varios tipos de productos obtenidos por rutas distintas, como es el caso de dos productos por vía seca y por vía húmeda, y también con espesores variados, ya que la base de cálculo es la masa cocida (en toneladas) y no la superficie.

El cálculo de las emisiones de dióxido de carbono se ha realizado siguiendo las directrices indicadas en la normativa europea sobre el comercio de emisiones (Directiva 2009/29/CE).

Las emisiones de $\mathrm{CO}_{2}$ procedentes de la combustión del gas natural se calcularon de acuerdo con el volumen total de gas natural consumido, el poder calorífico y el factor de emisión de combustible. Las emisiones por descomposición de los minerales carbonatados durante la cocción se calcularon de acuerdo con el contenido en carbonatos presentes en los diferentes tipos de productos y sus respectivos volúmenes de producción. Las emisiones de $\mathrm{CO}_{2}$ producidas por la combustión de la materia orgánica no se han considerado en este trabajo.

\section{RESULTADOS Y DISCUSIÓN}

\subsection{Consumo Térmico}

De acuerdo con un sondeo previo realizado en España y Brasil en los años 2008 y 2009, en ambos países el 92\% de la demanda final de energía necesaria para la fabricación de baldosas cerámicas se corresponde con demanda de energía térmica, como se muestra en la figura 2 [8 y 9]. Como el coste de la energía térmica es uno de los principales sobre el coste total de producción de baldosas cerámicas, conocer más profundamente como se consume la energía térmica consumida en las etapas de producción se convierte en una herramienta importante para las empresas.

En las tablas II, IV y VI se muestran los valores de consumo térmico de los atomizadores, secaderos y hornos estudiados, respectivamente. En cuanto a los atomizadores, se observa que los equipos de Brasil y España en general tienen un consumo térmico medio similar (tabla II). Sin embargo, en Brasil se observaron mayores discrepancias entre los consumos de los equipos, llegando a encontrarse diferencias de hasta el $40 \%$. Estas diferencias se observó que estaban relacionadas con el tipo de producto fabricado, y con el fabricante del atomizador [8].

En España, es habitual que los atomizadores utilicen como gases de secado los gases de escape de turbinas de gas. Las turbinas de gas son sistemas de cogeneración que, a partir de la combustión de gas natural, generan energía eléctrica y energía térmica. La energía eléctrica se consume en la propia planta productiva, y si hay excedentes se venden a la

\section{España y Brasil}

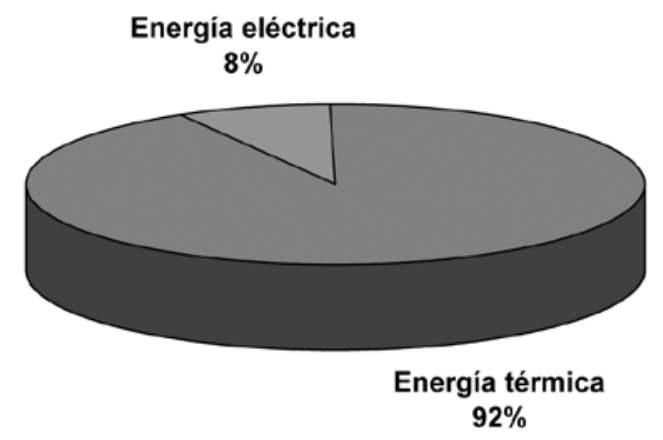

Figura 2. Distribución de consumos energéticos en el sector de fabricación de baldosas cerámicas y gránulo atomizado, durante el año 2008 (España) y 2009 (Brasil). 


\begin{tabular}{|c|c|c|c|}
\hline & & España & Brasil \\
\hline \multicolumn{2}{|c|}{ Número de atomizadores } & 12 & 11 \\
\hline \multirow{2}{*}{ Consumo específico * } & kWh/t sólido seco & $476 \pm 19$ & $\mathbf{5 0 2} \pm 87$ \\
\hline & kWh/t cocido & $510 \pm 23$ & $540 \pm 100$ \\
\hline
\end{tabular}

* el Poder Calorífico Superior (PCS) del gas natural comercializado en España y en Brasil es 39,83 MJ/Nm³ y 39,65 MJ/Nm³, respectivamente.

compañía eléctrica, que está obligada, por ley, a adquirirla. La energía térmica contenida en los gases de escape se utiliza en los atomizadores, que cuentan también con un quemador de postcombustión para terminar de cubrir las necesidades térmicas del atomizador cuando con los gases de la turbina no hay suficiente calor disponible para el proceso de secado. Típicamente una turbina de cogeneración produce una relación aproximada de 2 a 1 de energía térmica frente a energía eléctrica, en los cálculos de cada empresa se ha tenido en cuenta esta relación.

El consumo energético de los atomizadores en España, mostrado en la tabla II, corresponde a la suma de la energía aportada por los gases procedentes de la turbina de cogeneración y la energía proporcionada por el quemador de postcombustión.

En la tabla III se muestran las condiciones medias de operación de los atomizadores analizados en España y en Brasil. La diferencia más significativa se observa en el contenido en sólidos de la barbotina, pues en las suspensiones de Brasil es, en promedio, un $2 \%$ inferior al valor determinado en las suspensiones en España. Esta podría ser la causa del menor consumo energético de los atomizadores del sector español, respecto a los brasileños.

En la tabla IV se recogen los consumos de los secaderos según sus características constructivas (verticales u horizontales) y a la tecnología utilizada en la fabricación de las baldosas cerámicas (vía seca o vía húmeda). Asimismo, en la tabla V se muestran las condiciones medias de trabajo de los secaderos estudiados en España y en Brasil.

Los secaderos utilizados en las empresas españolas (vía húmeda) poseen menor consumo energético que los evaluados en Brasil. En España los secaderos verticales y horizontales estudiados presentan consumos térmicos parecidos. El mayor consumo de los secaderos del proceso vía seca puede estar asociado a una mayor humedad utilizada para el prensado en este tipo de productos en Brasil ( 9\%) y al mantenimiento de elevadas temperaturas $\left(200-320{ }^{\circ} \mathrm{C}\right)$ que son utilizadas para la eliminación rápida del agua en ciclos cortos 10 min (en secaderos horizontales), garantizando una elevada productividad. En Brasil las condiciones de secado en el proceso vía húmeda son más suaves que en el proceso vía seca: humedad para el prensado entre 5 y 6,5\%, temperatura de secado entre 150 y $200{ }^{\circ} \mathrm{C}$ y ciclos de 15 min (en secaderos horizontales). Las mayores discrepancias se observaron entre los consumos específicos de los secaderos del proceso vía seca, siendo posible encontrar equipos que consumían dos veces más de lo que lo hacían los más eficientes evaluados.

En los secaderos verticales del proceso vía húmeda, no se observan diferencias significativas entre los parámetros de secado analizados en ambos países. Sin embargo, el consumo energético en España es menor que en Brasil. Atendiendo a los secaderos horizontales, en España, a pesar de tener mayor duración y temperatura máxima el ciclo de secado, el consumo energético también es menor que en Brasil en estos

TABLA III. CONDICIONES MEDIAS DE OPERACIÓN EN LA ETAPA DE ATOMIZACIÓN.

\begin{tabular}{|c|c|c|}
\hline Parámetro & España & Brasil \\
\hline Producción de gránulo atomizado (t ss/h) & $23 \pm 4$ \\
\hline Contenido en sólidos de la barbotina (\%) & $65,0 \pm 0,7$ & $63,0 \pm 1,0$ \\
\hline Humedad del gránulo atomizado (\%) & $6,2 \pm 0,3$ & $6,0 \pm 0,5$ \\
\hline
\end{tabular}

TABla IV. CONSUMO DE ENERGÍA TÉRMICA EN LA ETAPA DE SECADO (REFERIDO AL PCS), EN ESTADO ESTACIONARIO.

\begin{tabular}{|c|c|c|c|c|c|c|c|}
\hline \multirow{3}{*}{\multicolumn{2}{|c|}{ Tipo de secadero }} & \multirow{2}{*}{\multicolumn{2}{|c|}{$\frac{\text { España }}{\text { Vía húmeda }}$}} & \multicolumn{4}{|c|}{ Brasil } \\
\hline & & & & \multicolumn{2}{|c|}{ Vía Húmeda } & \multicolumn{2}{|c|}{ Vía Seca } \\
\hline & & Verticales & Horizontales & Verticales & Horizontales & Verticales & Horizontales \\
\hline \multicolumn{2}{|c|}{ Número de equipos en el estudio } & 3 & 3 & 4 & 8 & - & 9 \\
\hline $\begin{array}{l}\text { Consumo } \\
\text { específico }\end{array}$ & kWh/t cocido & $\mathbf{1 3 0} \pm 19$ & $139 \pm 25$ & $153 \pm 18$ & $164 \pm 35$ & - & $232 \pm 90$ \\
\hline
\end{tabular}




\begin{tabular}{|c|c|c|c|c|c|c|}
\hline \multirow{2}{*}{ Parámetro } & \multicolumn{2}{|c|}{ España } & \multicolumn{3}{c|}{ Brasil } \\
\cline { 2 - 6 } & \multicolumn{2}{|c|}{ Vía húmeda } & Vía Húmeda & \multicolumn{2}{c|}{ Vía Seca } \\
\cline { 2 - 6 } & Verticales & Horizontales & Verticales & Horizontales & Verticales & Horizontales \\
\hline Producción (kg coc/s) & $2,2 \pm 0,3$ & $2,0 \pm 0,6$ & $2,4 \pm 0,3$ & $2,6 \pm 0,2$ & - & $3,0 \pm 0,2$ \\
\hline Humedad de entrada (\%) & $6,0 \pm 0,3$ & $6,0 \pm 0,1$ & $5,8 \pm 0,2$ & $5,8 \pm 0,2$ & - & $9,0 \pm 0,5$ \\
\hline Humedad de salida (\%) & $0,13 \pm 0,09$ & $0,5 \pm 0,3$ & $0,6 \pm 0,2$ & $0,7 \pm 0,3$ & - & $0,9 \pm 0,4$ \\
\hline $\begin{array}{c}\text { Duración del ciclo de secado } \\
\text { (min) }\end{array}$ & $77,9 \pm 22,4$ & $24,6 \pm 2,8$ & $80 \pm 8$ & $15 \pm 2$ & - & $10 \pm 1$ \\
\hline $\begin{array}{c}\text { Temperatura máxima } \\
\text { de secado }\left({ }^{\circ} \mathrm{C}\right)\end{array}$ & $172 \pm 19$ & $241 \pm 40$ & $160 \pm 15$ & $185 \pm 20$ & - & $270 \pm 40$ \\
\hline
\end{tabular}

secaderos. Esto puede ser debido a una diferente regulación en las instalaciones, lo que incide sobre la eficiencia del proceso de secado, dando lugar a las diferencias de consumo observadas [10].

En la tabla VI se resumen los valores de consumo térmico en la etapa de cocción, de los tipos de baldosas cerámicas más importantes para los sectores brasileño y español. En la tabla VII se muestran las características de cada tipo de producto evaluado en este trabajo y los parámetros de cocción utilizados.

En Brasil, se puede observar que la cocción de los productos con un proceso vía húmeda consume mayor energía que las de los productos por vía seca (tabla VI). Esto puede explicarse por el hecho de que los hornos estudiados que operan en los procesos por vía húmeda son de una generación más antigua que los utilizados en el proceso vía seca, lo que puede justificar un mayor consumo. Además, los productos producidos por vía húmeda poseen ciclos térmicos más largos que los producidos por vía seca y temperaturas máximas superiores, ya que las materias primas utilizadas son más refractarias (tabla VII). En general, las composiciones cerámicas de los procesos vía seca brasileñas están constituidas exclusivamente por arcillas rojas de carácter altamente fundente, que permiten la utilización de bajas temperaturas de cocción y ciclos muy rápidos (tabla VII). Sin embargo, cuando se compara el gres rojo con la monococción roja, ambos utilizando arcillas con propiedades parecidas, el mayor consumo térmico del gres rojo puede atribuirse a un ciclo térmico más largo y a mayores temperaturas de cocción, puesto que se debe alcanzar una menor porosidad.
En España, los resultados obtenidos muestran que, en general, el consumo específico medio de la cocción por unidad de masa de los productos porosos es mayor que el obtenido en productos gresificados. Esto es debido al hecho de que se produce la reacción de descomposición de los carbonatos presentes en la composición, que es endotérmica (es decir, requiere un aporte de energía), y esto ocasiona que los ciclos térmicos empleados para la cocción de productos porosos deban tener una etapa de precalentamiento con mucho aporte energético. En las composiciones de azulejos, son los productos formulados con arcillas de coloración blanca los que presentan mayor consumo, debido a los mayores tiempos de cocción que requieren estos productos.

En los productos gresificados, es el gres porcelánico el que presenta mayor consumo, por la mayor temperatura de cocción y duración del ciclo. Si estos mismos valores de consumo específico se expresan por metro cuadrado, se observa que el gres porcelánico es el producto que tiene mayor consumo, debido a su mayor peso específico medio.

Si se compara el consumo energético en la etapa de cocción de los distintos productos (tabla VI), obtenidos en los dos países por vía húmeda, se observa que el consumo energético específico de los productos cocidos en Brasil es menor que el de los mismos productos (azulejo blanco, gres rojo y gres porcelánico) obtenidos en España. Estas diferencias pueden deberse a la menor duración de los ciclos de cocción de estos productos en Brasil, tal y como se muestra en la tabla VII.

En la tabla VIII se resumen los resultados obtenidos en el estudio de consumo de energía térmica por etapas, para baldosas prensadas fabricadas por monococción. Se muestra

TABla VI. CONSUMO DE ENERGía téRMiCA EN LA ETAPA DE COCCIÓN (REFERIDO AL PCS), EN ESTADO ESTACIONARIO.

\begin{tabular}{|c|c|c|c|c|c|c|c|c|c|}
\hline \multicolumn{3}{|c|}{ Producto } & $\begin{array}{l}\text { Azulejo } \\
\text { rojo }\end{array}$ & $\begin{array}{l}\text { Azulejo } \\
\text { blanco }\end{array}$ & Gres rojo & $\begin{array}{l}\text { Gres por- } \\
\text { celánico }\end{array}$ & $\begin{array}{l}\text { Monococción } \\
\text { Blanca }\end{array}$ & $\begin{array}{c}\text { Monococción } \\
\text { Roja }\end{array}$ & $\begin{array}{c}\text { Total; } \\
\text { Valor medio }\end{array}$ \\
\hline \multirow{3}{*}{$\begin{array}{c}\text { Consumo } \\
\text { específico } \\
\text { (kWh/t } \\
\text { cocido) }\end{array}$} & \multicolumn{2}{|c|}{ España } & $816 \pm 27$ & $885 \pm 51$ & $724 \pm 18$ & $802 \pm 20$ & - & - & $793 \pm 14$ \\
\hline & \multirow{2}{*}{ Brasil } & $\begin{array}{c}\text { Vía } \\
\text { Húmeda }\end{array}$ & - & $688 \pm 99$ & $635 \pm 5$ & $705 \pm 45$ & $724 \pm 70$ & - & $683 \pm 73$ \\
\hline & & Vía Seca & - & - & - & - & - & $575 \pm 49$ & $575 \pm 49$ \\
\hline $\begin{array}{l}\text { Consumo } \\
\text { específico }\end{array}$ & \multicolumn{2}{|c|}{ España } & $13,4 \pm 0,5$ & $16,0 \pm 1,4$ & $14,3 \pm 0,4$ & $17,4 \pm 0,6$ & & & $15,5 \pm 0,4$ \\
\hline \multirow{2}{*}{$\begin{array}{l}\left(\mathbf{k W h} / \mathrm{m}^{2}\right. \\
\text { cocido) }\end{array}$} & \multirow{2}{*}{ Brasil } & $\begin{array}{c}\text { Vía } \\
\text { Húmeda }\end{array}$ & & $10,7 \pm 2,1$ & $\mathbf{9 , 5} \pm 0,3$ & $13,7 \pm 1,3$ & $10,9 \pm 1,8$ & - & $\mathbf{1 2 , 1} \pm 0,7$ \\
\hline & & Vía Seca & & & & & & $8,3 \pm 1,2$ & $8,3 \pm 1,2$ \\
\hline
\end{tabular}


tanto el intervalo de variación como el valor medio de consumo en cada una de las etapas estudiadas. Todos los valores se han referido a toneladas de producto cocido, de forma que es posible realizar la adición directa de los consumos en las distintas etapas para calcular el consumo total. Asimismo, en la misma tabla se indican los intervalos de consumo de energía térmica presentados en el BREF de fabricación de productos cerámicos para cada una de las etapas del proceso y el punto central de dicho intervalo [11].

De acuerdo con la tabla VIII el consumo térmico medio de los procesos vía seca se debe en un $71 \%$ a la etapa de cocción y un $29 \%$ a la etapa de secado, lo que indica que el consumo de los hornos es aproximadamente 2,5 veces mayor que el de los secaderos. En el proceso vía húmeda la etapa de atomización es la responsable de un $39 \%$ del consumo térmico total y los hornos presentan un consumo 4,3 veces mayor que el de los secaderos.

En la etapa de atomización, los intervalos de variación de los consumos obtenidos en los estudios realizados en España y Brasil son menores que el indicado por el BREF, si bien el valor medio de consumo en España es similar al punto central indicado por el BREF y en Brasil es superior.

En las etapas de secado y cocción la amplitud de los intervalos de consumos obtenidos en los estudios experimentales es menor que el intervalo recogido en el BREF, y el valor medio del consumo en ambas etapas en los dos países estudiados es inferior al valor del punto central calculado a partir del intervalo indicado en el BREF.

El consumo global de energía en los procesos vía húmeda analizados en España y en Brasil es similar (1428 y 1383 $\mathrm{kWh} / \mathrm{t}$ cocido, respectivamente); la diferencia encontrada puede justificarse por el menor consumo específico de los hornos evaluados en Brasil, que se relaciona a su vez con la utilización de ciclos térmicos más cortos debido a la obtención de productos con espesores menores (tabla VII) y materias primas más fundentes. Sin embargo, el consumo global del proceso vía seca desarrollado en Brasil es muy inferior (807 $\mathrm{kWh} / \mathrm{t}$ cocido, sobre un $42 \%$ menor) al consumo medio de los procesos vía húmeda. No obstante, los valores deben tomarse con prudencia, puesto que el proceso vía seca desarrollado en Brasil presenta algunas limitaciones técnicas, por lo que se utiliza únicamente para la producción de un único producto (monococción roja) de formatos inferiores a $55 \times 55 \mathrm{~cm}^{2}$, que tiene una elevada porosidad en cocido (absorción de agua comprendida entre 6,0 y $10 \%$ ).

Estos datos indican que los sectores español y brasileño de fabricación de baldosas cerámicas utilizan las Mejores

TAbla VII. PARÁmetros de FUnCIONAMIENTO MEDiOS EN LA ETAPA DE COCCIÓN DE BALDOSAS CERÁMICAS PRENSADAS EN HORNOS DE RODILLOS, EN ESTADO ESTACIONARIO.

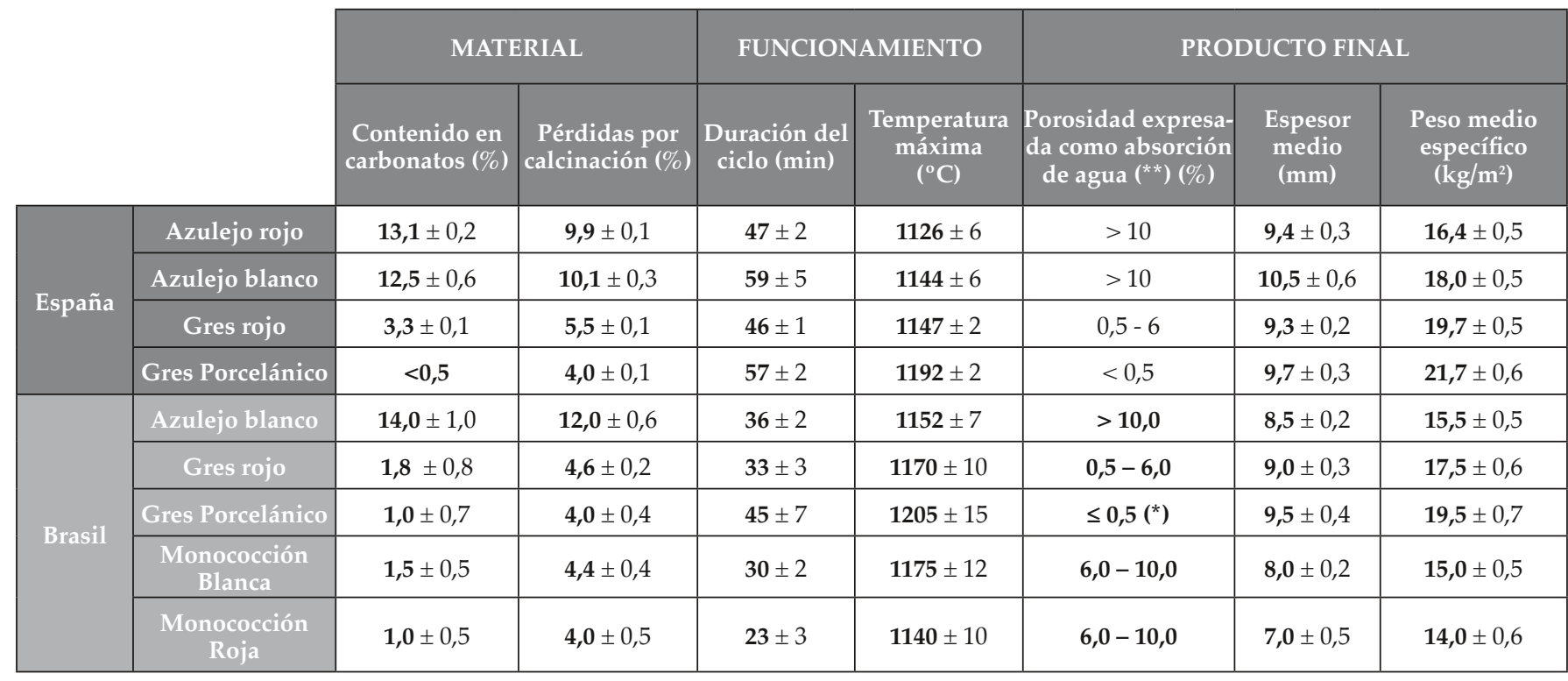

(*) en Brasil, de acuerdo con la normativa NBR 15463/2007 el gres porcelánico técnico (pulido) debe tener absorción de agua $\leq 0,1$.

$\left.{ }^{* *}\right)$ Intervalos de absorción de agua para clasificar los productos, según norma ISO 13006.

TABLA VIII. CONSUMOS DE ENERGÍA TÉRMICA OBTENIDOS Y COMPARACIÓN CON EL BREF (TODOS LOS VALORES ESTÁN EXPRESADO EN kWh/t COCIDO Y REFERIDOS AL PCS).

\begin{tabular}{|c|c|c|c|c|c|c|c|c|}
\hline & \multirow{2}{*}{\multicolumn{2}{|c|}{ Estudio del sector español }} & \multicolumn{4}{|c|}{ Estudio del sector brasileño } & \multirow{2}{*}{\multicolumn{2}{|c|}{ BREF }} \\
\hline & & & \multicolumn{2}{|c|}{ Vía Húmeda } & \multicolumn{2}{|c|}{ Vía Seca } & & \\
\hline & Intervalo & Valor medio & Intervalo & Valor medio & Intervalo & Valor medio & Intervalo & Punto central ${ }^{()}$ \\
\hline Atomización & $387-621$ & $510 \pm 23$ & $375-637$ & $540 \pm 100$ & - & - & $339-679$ & 509 \\
\hline Secado & $97-160$ & $125 \pm 20$ & $126-236$ & $160 \pm 30$ & $135-444$ & $232 \pm 90$ & $92-247$ & 170 \\
\hline Cocción & $578-1182$ & $793 \pm 14$ & $605-892$ & $683 \pm 73$ & $522-647$ & $575 \pm 49$ & $586-1480$ & 1033 \\
\hline Total & $1062-1963$ & $1428 \pm 57$ & $1106-1765$ & $1383 \pm 203$ & $657-1091$ & $807 \pm 139$ & $1017-2406$ & 1712 \\
\hline
\end{tabular}

$\left(^{*}\right)$ El punto central se ha calculado como la media aritmética de los extremos del intervalo. 


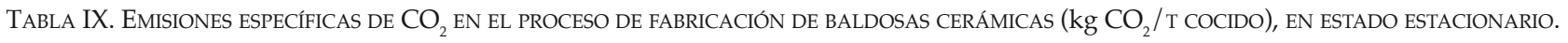

\begin{tabular}{|c|c|c|c|c|c|c|c|c|c|c|}
\hline \multirow{3}{*}{\multicolumn{2}{|c|}{ Etapa del proceso }} & \multicolumn{3}{|c|}{ Emisiones de combustión } & \multicolumn{3}{|c|}{ Emisiones de proceso } & \multicolumn{3}{|c|}{ TOTAL } \\
\hline & & \multirow[b]{2}{*}{ España* } & \multicolumn{2}{|c|}{ Brasil $^{* *}$} & \multirow[b]{2}{*}{ España* } & \multicolumn{2}{|c|}{ Brasil ** $^{* *}$} & \multirow[b]{2}{*}{ España* } & \multicolumn{2}{|c|}{ Brasill ** $^{* *}$} \\
\hline & & & $\begin{array}{c}\text { Vía } \\
\text { Húmeda }\end{array}$ & $\begin{array}{l}\text { Vía } \\
\text { Seca }\end{array}$ & & $\begin{array}{c}\text { Vía } \\
\text { Húmeda }\end{array}$ & $\begin{array}{l}\text { Vía } \\
\text { Seca }\end{array}$ & & $\begin{array}{c}\text { Vía } \\
\text { Húmeda }\end{array}$ & $\begin{array}{l}\text { Vía } \\
\text { Seca }\end{array}$ \\
\hline & nización & $93 \pm 4$ & $99 \pm 5$ & - & - & - & - & $93 \pm 4$ & $99 \pm 5$ & - \\
\hline & ecado & $23 \pm 2$ & $29 \pm 2$ & $42 \pm 4$ & - & - & - & $23 \pm 2$ & $29 \pm 2$ & $42 \pm 4$ \\
\hline \multirow{6}{*}{ Cocción } & Azulejo rojo & $149 \pm 5$ & - & - & $64 \pm 1$ & - & - & $213 \pm 5$ & - & - \\
\hline & Azulejo blanco & $161 \pm 9$ & $126 \pm 11$ & - & $61 \pm 3$ & $70 \pm 3$ & - & $222 \pm 10$ & $196 \pm 12$ & - \\
\hline & Gres rojo & $132 \pm 3$ & $116 \pm 2$ & - & $15 \pm 1$ & $8 \pm 2$ & - & $147 \pm 3$ & $124 \pm 2$ & - \\
\hline & $\begin{array}{c}\text { Gres } \\
\text { Porcelánico } \\
\end{array}$ & $147 \pm 4$ & $129 \pm 5$ & - & $<1$ & $5 \pm 1$ & - & $147 \pm 4$ & $134 \pm 5$ & - \\
\hline & $\begin{array}{c}\text { Monococción } \\
\text { roja } \\
\text { (esmaltada) } \\
\end{array}$ & - & - & $105 \pm 6$ & - & - & $5 \pm 1$ & - & - & $110 \pm 6$ \\
\hline & $\begin{array}{c}\text { Monococción } \\
\text { blanca } \\
\text { (esmaltada) }\end{array}$ & - & $132 \pm 8$ & - & - & $7 \pm 1$ & - & - & $139 \pm 8$ & - \\
\hline \multirow{6}{*}{ TOTAL } & Azulejo rojo & $265 \pm 11$ & - & - & $64 \pm 1$ & - & - & $329 \pm 11$ & - & - \\
\hline & Azulejo blanco & $277 \pm 15$ & $254 \pm 18$ & - & $61 \pm 3$ & $70 \pm 3$ & - & $338 \pm 18$ & $324 \pm 20$ & - \\
\hline & Gres rojo & $248 \pm 9$ & $244 \pm 9$ & - & $15 \pm 1$ & $8 \pm 2$ & - & $263 \pm 9$ & $252 \pm 9$ & - \\
\hline & $\begin{array}{c}\text { Gres } \\
\text { Porcelánico }\end{array}$ & $263 \pm 10$ & $257 \pm 12$ & - & $<1$ & $5 \pm 1$ & - & $263 \pm 10$ & $262 \pm 12$ & - \\
\hline & $\begin{array}{c}\text { Monococción } \\
\text { roja } \\
\text { (esmaltada) } \\
\end{array}$ & - & - & $147 \pm 10$ & - & - & $5 \pm 1$ & - & - & $152 \pm 10$ \\
\hline & $\begin{array}{c}\text { Monococción } \\
\text { blanca } \\
\text { (esmaltada) }\end{array}$ & - & $260 \pm 15$ & - & - & $7 \pm 1$ & - & - & $267 \pm 15$ & - \\
\hline
\end{tabular}

* en España, el factor de emisión del gas natural es de $202 \mathrm{~g} \mathrm{CO}_{2} / \mathrm{kWh}$

** en Brasil, el factor de emisón del gas natural es de $183 \mathrm{~g} \mathrm{CO}_{2} / \mathrm{kWh}$
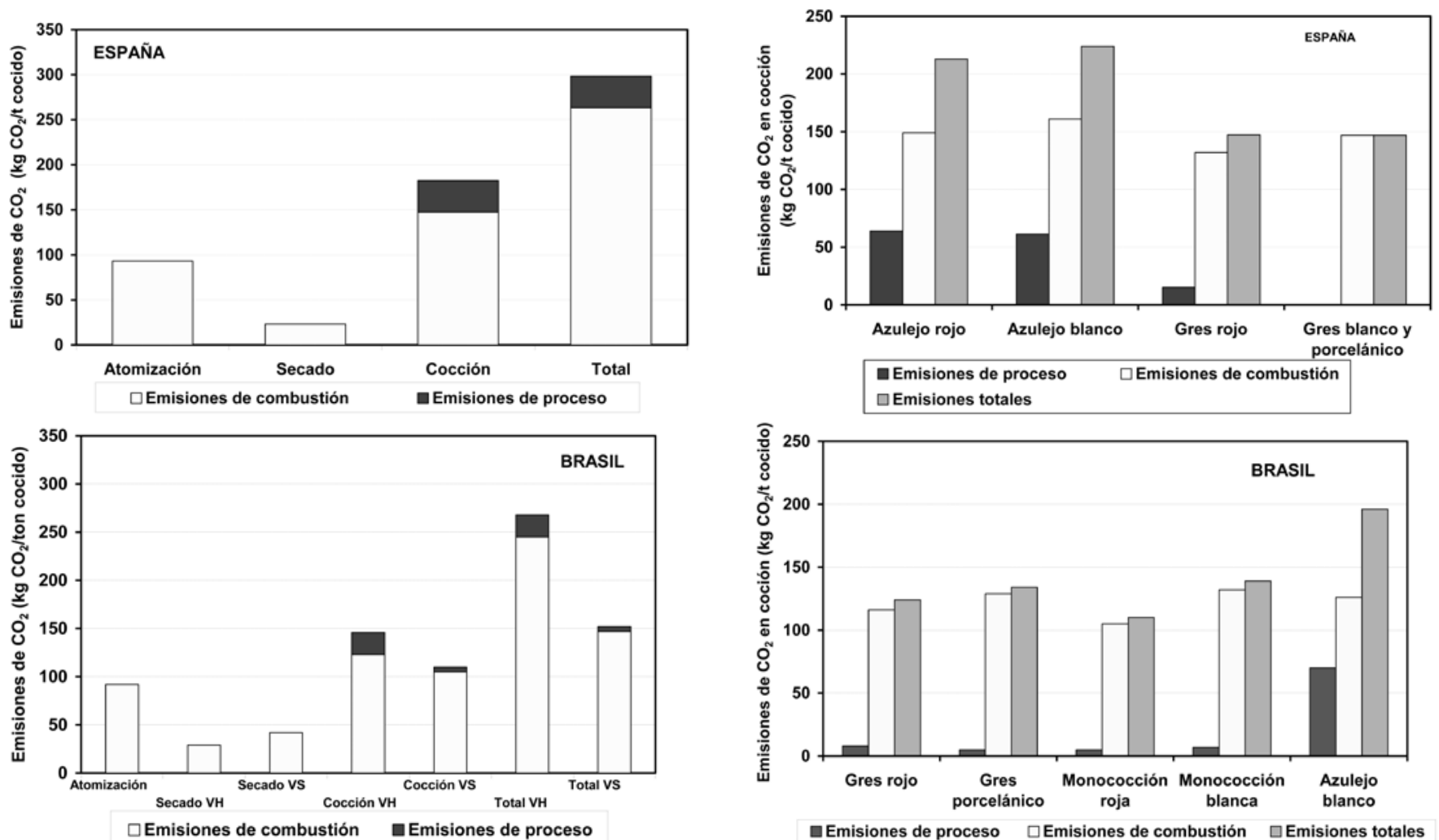

VH: proceso vía húmeda

VS. Proceso vía seca

Figura 3. Emisiones medias de $\mathrm{CO}_{2}$ (estado estacionario).

Figura 4. Emisiones de $\mathrm{CO}_{2}$ en la etapa de cocción (estado estacionario) 
TABLA X. EMISIONES ESPECíficAS DE $\mathrm{CO}_{2}$ EN EL PROCESO DE FABRICACIÓN DE BALDOSAS CERÁMICAS.

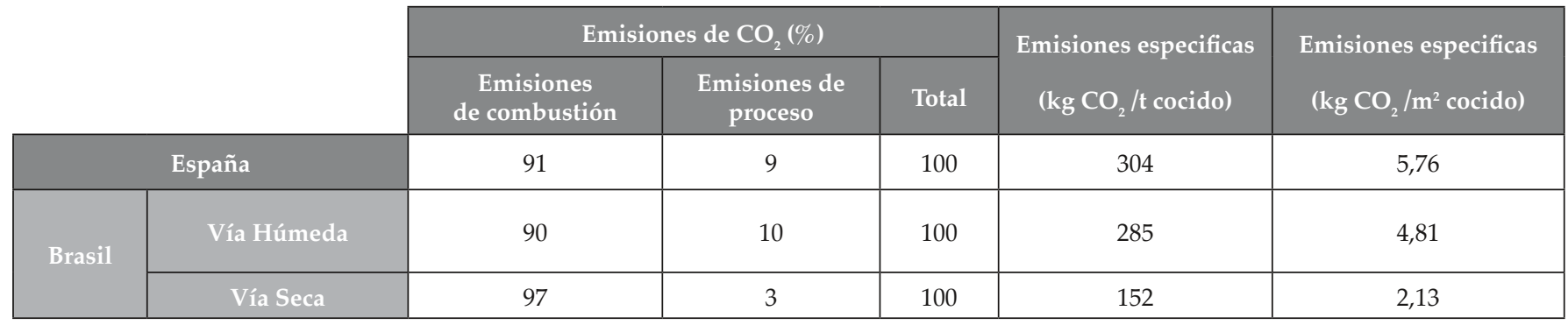

Tecnologías Disponibles, y que realizan una gestión eficaz de estas tecnologías [12], por lo que la capacidad de reducción del consumo y de las emisiones por unidad de producto a corto plazo está limitada y debe basarse en aplicar medidas de optimización de proceso y de ahorro energético [13 y 14].

\subsection{Emisiones de $\mathrm{CO}_{2}$}

En la tabla IX se presentan las emisiones medias de $\mathrm{CO}_{2}$ procedentes de la fabricación de baldosas cerámicas en España y en Brasil. Los datos representados en la figura 3 corresponden a las emisiones resultantes de la combustión de gas natural en cada etapa del proceso, en estado estacionario. Las emisiones generadas en la etapa de cocción (emisiones de combustión y de proceso) se han organizado por tipo de producto, como se puede observar en la figura 4.

En general, las emisiones específicas medias de $\mathrm{CO}_{2}$ en la etapa de cocción que provienen de la obtención de los mismos productos (azulejo blanco, gres rojo y gres porcelánico) por un proceso vía húmeda en Brasil y en España son inferiores en Brasil, debido principalmente a la menor duración de los ciclos en Brasil. En el caso del gres rojo, además, el contenido en carbonatos de las composiciones empleadas en España es mayor que en Brasil. A su vez, las emisiones resultantes de la producción de baldosas cerámicas por vía seca son mucho menores que por vía húmeda, porque por vía seca los ciclos térmicos utilizados en la cocción de los productos son más cortos y las materias primas tienen bajos niveles de carbonatos, lo que implica menores niveles de emisiones de combustión y de proceso, respectivamente (tabla IX y figura 3 ).

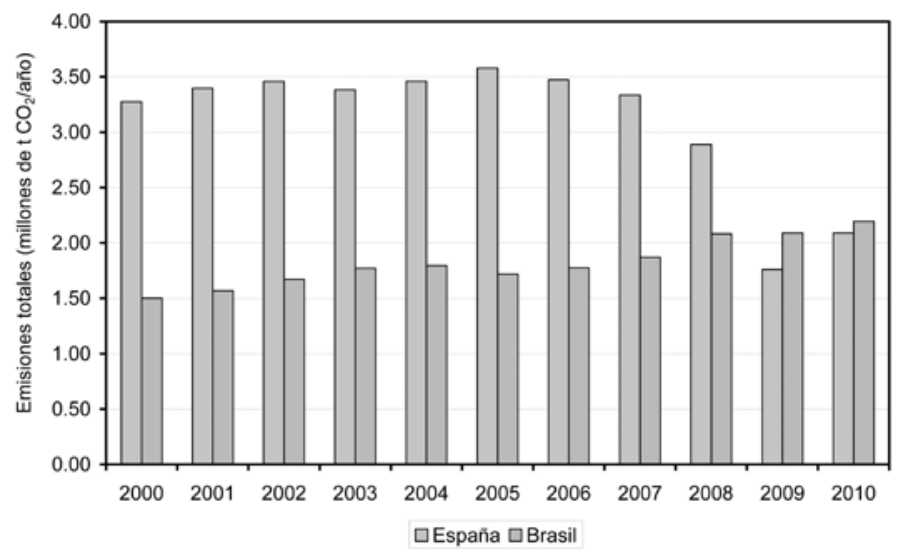

Figura 5. Evolución de las emisiones sectoriales de $\mathrm{CO}_{2}$ en España y en Brasil.
Según los datos presentados en la tabla $X$, las emisiones específicas medias producidas en la producción de baldosas cerámicas por vía húmeda en España y Brasil son similares. Las emisiones específicas referidas a masa de producto cocido entre España y Brasil difieren un 6\%, mientras que las emisiones referidas a unidad de superficie ascienden al $16 \%$. Esta diferencia en las emisiones específicas referidas a unidad de superficie se debe a la diversidad de formatos fabricados por España y a los mayores formatos fabricados.

Dado que el proceso vía seca, por prescindir de la etapa de atomización, utiliza ciclos de cocción más cortos y materias primas con niveles más bajos de carbonatos, emite a la atmósfera casi el $50 \%$ menos de $\mathrm{CO}_{2}$ por tonelada de producto que cuando se utiliza el proceso vía húmeda.

La evolución de las emisiones totales en la última década en los sectores español y brasileño se ha representado en la figura 5. Se aprecia cómo, en España, dado que no ha habido ningún cambio tecnológico de relevancia en el proceso productivo en el periodo de tiempo considerado, la evolución de las emisiones es paralela a la de la producción nacional. En Brasil, el aumento progresivo de la producción en la última década ha sido el principal factor responsable del aumento de las emisiones. Sin embargo, a pesar de que la producción nacional ha aumentado en más del $65 \%$ en los últimos 10 años (figura 1), como fueron los productos producidos por vía seca los que impulsaron este crecimiento, el incremento de las emisiones totales fue inferior al $46 \%$ en el mismo periodo (figura 5), ya que los productos producidos por vía seca tienen una emisión específica menor.

\section{CONCLUSIONES}

Las principales conclusiones del trabajo realizado son las siguientes:

- En los dos países analizados, España y Brasil, el 92\% de la demanda final de energía del sector de fabricación de baldosas cerámicas y gránulo atomizado, corresponde al consumo de energía térmica, mientras que el consumo de energía eléctrica supone el $8 \%$ del consumo energético total.

- El consumo energético medio de los atomizadores en Brasil es superior al de los atomizadores en España, esa diferencia parece deberse al menor contenido en sólidos de las suspensiones utilizadas en Brasil. Por otra parte, cabe destacar que la fabricación de gránulo atomizado en España suele realizarse con aporte de calor procedente de sistemas de cogeneración, mediante 
turbinas, y en caso de requerirse, se aporta calor adicional mediante quemadores de post-combustión. En Brasil ninguna de las instalaciones estudiadas utiliza sistemas de cogeneración. Por ello, la eficiencia energética global (eléctrica y térmica) en la industria española es superior, ya que los sistemas de cogeneración mediante turbinas de gas presentan un rendimiento global del orden del $90 \%$.

- Los secaderos verticales y horizontales empleados en el proceso vía húmeda de Brasil muestran un mayor consumo energético que los de España, a pesar de que los parámetros de trabajo son similares. Las diferencias encontradas podrían deberse a diferentes formas de regular los secaderos que inciden de manera distinta sobre la eficiencia del proceso. En la industria brasileña, los secaderos utilizados en el proceso vía seca tienen un mayor consumo que los utilizados en el proceso vía húmeda, debido a la mayor humedad de entrada de los soportes a secar, y a la mayor temperatura máxima de secado.

- El consumo de la etapa de cocción en el proceso con molienda vía húmeda, es inferior en Brasil que en España, probablemente debido a la mayor fundencia de las materias primas empleadas, que posibilitan el empleo de ciclos de cocción más cortos. Las materias primas utilizadas en el proceso vía seca en Brasil son muy fundentes, y permiten el empleo de ciclos de cocción muy cortos, por lo que el consumo energético de los hornos cuando se fabrica este producto (monococción roja) es inferior al de los hornos que procesan otros productos obtenidos por vía húmeda, aunque este producto presenta limitaciones técnicas.

- Los valores de consumos energéticos medios por etapas obtenidos en los estudios realizados se sitúan en el intervalo indicado en el documento BREF de la Comisión Europea. Se concluye que las industrias cerámicas española y brasileña están utilizando de forma generalizada las Mejores Técnicas Disponibles. En la situación actual, se puede realizar una reducción de consumos significativa a nivel individual aplicando medidas de optimización de proceso y de ahorro energético, a pesar de que los márgenes de reducción a nivel sectorial parecen relativamente estrechos en ambos países. Una reducción significativa de las emisiones sectoriales sólo parece posible con cambios tecnológicos importantes en los equipos consumidores de energía térmica y/o con la sustitución de las actuales fuentes de energía.

- En general, las emisiones específicas de $\mathrm{CO}_{2}$ en el procesado vía húmeda de baldosas cerámicas en España y Brasil, asociados al proceso de fabricación son similares, aunque en la etapa de cocción se aprecian algunas diferencias debidas principalmente a la menor duración de los ciclos de cocción en Brasil. El proceso vía seca utilizado en Brasil presenta menores emisiones debido a la ausencia del secado por atomización, al menor contenido en carbonatos de las materias primas utilizadas, y a la menor duración de los ciclos de cocción, pero el producto obtenido por este procedimiento tiene unas limitaciones técnicas que restringen su uso a determinadas aplicaciones.
- Las principales ventajas competitivas encontradas en materia de consumo de energía y emisiones de $\mathrm{CO}_{2}$ en los dos países son: i) en Brasil, la elevada producción de productos fabricados por vía seca, que a pesar de algunas limitaciones técnicas, presentan menores consumos y emisiones de $\mathrm{CO}_{2}$ específicas; y ii) en España, la elevada optimización a la que operan los secaderos por atomización, junto con el uso generalizado de sistemas de cogeneración, lo que permite aumentar la eficiencia energética global (eléctrica y térmica) del proceso.

\section{AGRADECIMIENTOS}

El estudio presentado ha sido financiado por el Instituto de la Mediana y Pequeña Industria Valenciana (IMPIVA) dentro del Programa de investigación y Desarrollo Tecnológico a través del proyecto IMDEEA/2011/109.

\section{REFERENCIAS BIBLIOGRÁFICAS}

[1] Giacomini, P. World production and consumption of ceramic tiles. Ceramic world review, 88, (2010), 52-68.

[2] Crasta, G. P. Costi e ricavi dell'industria ceramica italiana. Ceramic world review, 65(16), (2006), 46-50.

[3] Monfort, E.; Mezquita, A.; Granel, R.; Vaquer, E.; Escrig, A.; Miralles, A.; Zaera, V. Análisis de consumos energéticos y emisiones de dióxido de carbono en la fabricación de baldosas cerámicas. Boletín de la Sociedad Española de Cerámica y Vidrio, 49(4) (2010), 303-310.

[4] Directiva 2003/87/CE del Parlamento Europeo y del Consejo, del 13 de octubre de 2003, por la que se establece un régimen para el comercio de derechos de emisión de gases de efecto invernadero en la Comunidad y por la que se modifica la Directiva 96/61/CE del Consejo.

[5] Directiva 2009/29/CE del Parlamento Europeo y del Consejo, de 23 de Abril de 2009, por la que se modifica la Directiva 2003/87/CE, para perfeccionar y ampliar el régimen comunitario de comercio de derechos de emisión de gases de efecto invernadero.

[6] Mezquita, A.; Monfort, E.; Zaera, V. Sector azulejero y comercio de emisiones: reducción de emisiones de CO2, benchmarking europeo. Boletín de la Sociedad Española de Cerámica y Vidrio. 48(4) (2009), 211-222.

[7] ANFACER - Associação Nacional de Fabricantes de Cerâmica de Revestimento. Disponível em www.anfacer.org.br. Data da consulta: $15 / 04 / 2011$.

[8] Alves, H. J.; Melchiades, F. G.; Boschi, A. O. Consumo de gás natural na indústria de revestimentos cerâmicos brasileira. Cerâmica, 54, (2008), 326331.

[9] Alves, H. J.; Melchiades, F. G.; Boschi, A. O. Levantamento inicial do consumo de energias térmica e elétrica na indústria brasileira de revestimentos cerâmicos. Cerâmica Industrial, 12(1/2), (2007), 17-21.

[10] Mallol, G.; Mezquita, A.; Llorens, D.; Jarque, J.C.; Sahún, J; Valle, F. Estudio de la operación de secado de los soportes de las baldosas cerámicas en secaderos verticalesCerámica Información, $\mathrm{n}^{\circ}$ 287, págs, 81-93, 2002.

[11] Reference Document on Best Available Techniques in the ceramic manufacturing industry. Sevilla: European comission. European IPPC Bureau, 2007 http: / / ftp.jrc.es / eippcb / doc/cer_bref_0807.pdf [Consulta: 2009-06-25].

[12] Escardino, A. El esfuerzo en innovación de la industria cerámica de la Comunidad Valenciana para reducir las emisiones de dióxido de carbono. EN: Simposio internacional sobre el cambio climático, desde la ciencia a la sociedad. Valencia: Generalitat Valenciana, 2005. pp. 121-133.

[13] Monfort, E.; Mallol, G.; Mezquita, A.; Granel, R.; Vaquer, E. Estudio de la combustión en hornos industriales de fabricación de baldosas cerámicas. Póster presentado en el XI Congreso Mundial de la Calidad del Azulejo y del Pavimento Cerámico (Febrero 2010).

[14] Enrique, J.E.; Blasco, A.; Monfort, E.; Mallol, G. Improving energy efficiency in single-deck kilns by optimization of the process variables. Cfi Ber. DKG, 72(5), 255-260, 1995. 